\title{
INVESTIGACIÓN ACERCA DE \\ LA PRESENCIA O AUSENCIA DE AUTOCORRELACIÓN DE TEMPERATURA Y SALINIDAD EN PROFUNDIDADES ESTÁNDAR HASTA LOS 500 METROS
}

Elaborado por: Igor Málikov, Msc. en Ing. Oceanológica.

\section{Resumen}

Por medio del presente estudio se han determinado capas y zonas del Pacífico Colombiano con diferentes condiciones físicas según el coeficiente de correlación para las características de Temperatura y Salinidad.

Los resultados obtenidos no se pueden considerar acertados en su totalidad ya que para conseguir el cálculo de los coeficientes de correlación se utilizaron las características medias mensuales de Temperatura y Salinidad obtenidas a través de una insuficiente cantidad de datos iniciales, lo cual disminuye la veracidad de dichos promedios.

Los resultados presentados en este trabajo muestran una visión aproximada de las dependencias estadísticas entre las profundidades analizadas con respecto a la Temperatura y Salinidad.

\section{Abstract}

The present study makes possible to determine Colombian Pacific Ocean layers and regions with different physical conditions according to correlation coefficients for temperature and salinity. Results obtained are not accurate at all because in the end to calculate the correlation coefficients, it was necessary to make use of temperature and salinity monthly average values achieved with insufficient quantity of data. Results presented in the paper show an approximate view of the staditic dependence between parameters values at reference depths. 


\section{INTRODUCCIÓN}

Para la investigación de procesos o fenómenos hidrometeorológicos, es necesario estudiar las relaciones entre diferentes parámetros. Para poder modelar la dinámica del Pacífico Colombiano se deben determinar las posibles relaciones entre parámetros hidrológicos.

Existen muchos métodos de determinación de relaciones entre los parámetros, de los cuales más popular es el Coeficiente de Correlación, con el cual se definen las relaciones en forma lineal. Esto método fue utilizado en el presente trabajo. La búsqueda se realizó para cada parámetro entre las profundidades estándar, ello permitió diferenciar unas zonas y capas dentro del Pacífico Colombiano, las cuales presentan cambios entreanuales similares entre sí.

\section{DATOS UTILIZADOS}

En el presente trabajo fueron utilizados los valores promedios mensuales de Temperatura y Salinidad de 82 estaciones en la región de estudio (Fig. No.1).

Cada estación tiene 12 profundidades estándar (de 0 a 500 metros) y cada profundidad posee características mensuales en un año de variaciones en Temperatura y Salinidad.

Estos datos se obtuvieron por medio de una aproximación polinomial (Málikov, 1998) de los datos iniciales de los cruceros sobre el Pacífico Colombiano durante el periodo 1970 - 1996.

\section{OBJETIVO GENERAL}

Buscar las posibles relaciones estadísticas entre las profundidades estándar por medio de la Temperatura y la Salinidad del mar en cada estación de la región de estudio.

\section{METODOLOGÍA}

En el tiempo actual se conocen diferentes indicadores de las relaciones estadísticas entre dos series de datos. El mas conocido es el coeficiente de correlación, el cual representa la característica de una relación lineal de valores aleatorios $X$ y $Y$ no dependiendo de sus unidades de medida, o sea:
(1)

$$
r=\frac{\sum_{i=1}^{n}\left(X_{i}-\bar{X}\right)\left(Y_{i}-\bar{Y}\right)}{n \sigma_{X} \sigma_{Y}}
$$

Donde, $r$ es el Coeficiente de Correlación;

$\sigma=\sqrt{\frac{\sum_{i=1}^{n}\left(X_{i}-\bar{X}\right)^{2}}{n}}, \begin{gathered}\text { promedio de desviación } \\ \text { cuadrado; }\end{gathered}$

$\bar{X}$ - promedio de la serie ;

$\bar{Y}$ - promedio de la serie.

Para las series cortas, el denominador de la expresión (1) tendrá el coeficiente $(n-1)$ en lugar de $n$.

Algunas propiedades importantes del coeficiente de correlación son:

1- Su valor no cambia si se aumenta o disminuye a los valores X y Y un valor constante.

2- Su valor no cambia si se divide o multiplica a los valores $X$ y $Y$ un valor positivo cualquiera.

De la formula (1) se obtiene, que el coeficiente de correlación varia entre los limites $-1 \leq r \leq 1$.

Si $r= \pm 1$, entonces, la relación entre los elementos $X$ y $Y$ es funcional. En este caso, los datos en una gráfica de relación se representaran por medio de una línea inclinada por un ángulo con respecto al eje de coordenadas de $X$.

Si $r= \pm 1>0$, entonces la relación entre los elementos $X$ y $Y$ es directamente proporcional, o sea que los dos valores relacionados aumentan o disminuyen al mismo tiempo.

Si $r<0$, entonces la relación entre los elementos $\mathrm{X}$ y $Y$ no es directamente proporcional, o sea, que al aumentar uno de estos valores, el otro disminuye.

Cuando los valores $\mathrm{X}$ y $\mathrm{Y}$ son independientes en el sentido estadístico, entonces $r=0$.

La distribución de los coeficientes de correlación elegidos $r^{*}$ depende mucho del tamaño de las series comparadas y del valor $r^{*}$.

Si el valor de $r^{*}$ es pequeño y la cantidad de datos es mucha, la distribución de $r^{*}$ cumple con una ley denominada "distribución normal". En este 


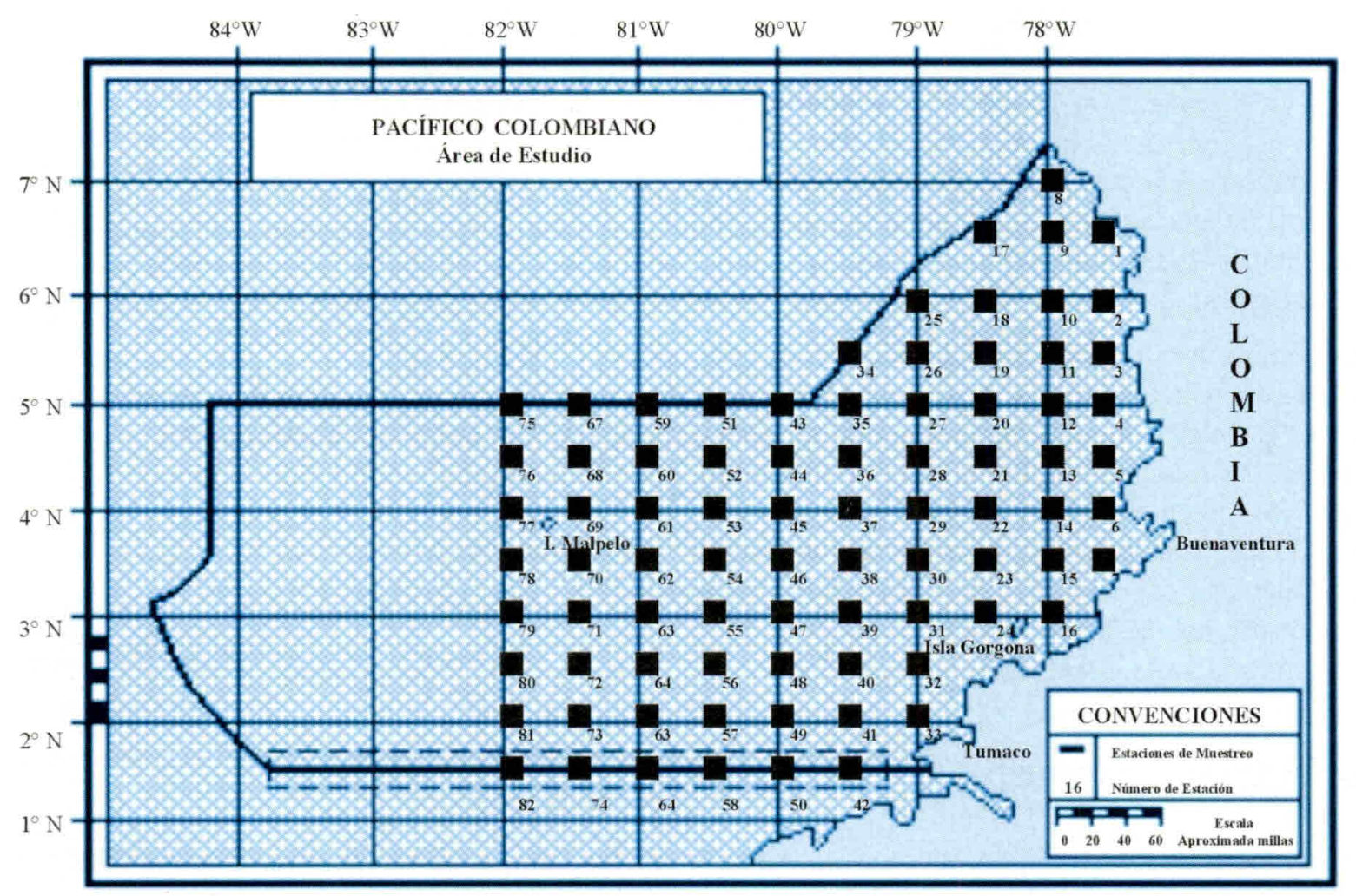

Fig. No. 1

caso, para encontrar errores casuales, se utiliza el método común de parametrizacion con pruebas de hipótesis.

Con el aumento de $r^{*}$ y disminución del volumen de datos $(n)$, la distribución de $r$ toma una forma de carácter no simétrico. Por eso, se hace necesario la úílización de métodos especializados para valorar la veracidad de $r^{*}$.

Para valorar la veracidad de los coeficientes de correlación elegidos $r^{*}$ ante diversos $n$, se utilizan las formulas de Romanov o de Fisher (con la distribución del coeficiente de stiudenta), etc.

Así, con la ayuda de esta metodología se realizo un análisis de la obtención de relaciones estadísticas entre las profundidades estándar de cada estación de la región de estudio. O sea, cada profundidad fue revisada por medio de relaciones estadísticas con las demás profundidades, por ejemplo: la profundidad 0 metros con la de 10 metros, $20, \ldots, 500$ metros; luego, la profundidad $10 \mathrm{me}-$ tros con la 20, 30, ..., 500 metros y así sucesivamente.

\section{RESULTADOS}

Los resultados obtenidos de la búsqueda de correlación entre las profundidades estándar se presentan en forma de mapas. En dichos mapas se determinan las regiones por colores diferentes y por capas, entre las cuales existen relaciones estadísticas.

Se muestran 4 mapas, dos de los cuales representan a la Temperatura (Fig. No.2 y 3) y dos a la Salinidad (Figs. No.4 y 5). Esto debido a que la capa de agua estudiada (0 - 500 metros) se dividió en dos partes: superficial y profunda, ya que en medio de ellas no se observo una relación y solo dentro de cada una de estas dos partes se encontraron dependencias.

En la Figura No.2 se observan las regiones de distribución estadística entre las capas superficiales con respecto a la Temperatura. La capa superficial más delgada del Pacifico Colombiano se encuentra en la región cercana a la isla Malpelo. Se denomino "delgada" debido a que existe dependencia estadistica en el parametro "temperatura" solamente entre las dos primeras profundidades (0 y 10 metros). Luego, le sigue una capa menos delgada (0-30 metros), la cual se localiza a lo largo de toda la costa del Pacifico 
Colombiano y también los sectores sur y suroccidental. Gran parte de las aguas del Pacifico Colombiano esta constuida por una capa de 0-50 metros. Dentro de esta capa, en la zona limitada por $78^{\circ}-80^{\circ} \mathrm{W}$ y $4^{\circ}-5^{\circ} \mathrm{N}$ se observa una pequeña región donde no existe una relación estadística entre las capas superficiales. En el norte del Pacifico Colombiano desde los $5^{\circ} \mathrm{N}$ y más, se determina un sector de dependencia entre la capa 0-100(150) metros.

Entre las capas "profundas" (Fig. No.3), se observa que la capa más delgada (20-100 metros) también se encuentra en los alrededores de la isla Malpelo. Luego, la capa mas gruesa correspondiente a los 100-200(300) metros, se encuentra en tres regiones alejadas entre sí: la primera al norte de la isla Malpelo, la segunda al sur del Pacifico Colombiano entre $81^{\circ} \mathrm{W}$ y $2^{\circ} \mathrm{N}$, y la tercera, la cual es más amplia, se localiza a lo largo de toda la costa del Pacifico Colombiano.

La capa de 100-500 metros se ubica en los sectores restantes de la región de estudio.

Se observan también dos sectores (del mismo color del agua del Pacifico Colombiano) en los cuales no existen relaciones estadísticas entre las profundidades.

En cuanto a los resultados de la correlación entre profundidades con respecto a la Salinidad, se tiene la Figura No.4 donde se representan a las capas superficiales: la capa más delgada 0-20(30) metros se observa a lo largo de la costa y se distribuye desde la costa hacia la parte norte del Pacifico Colombiano $\left(80^{\circ} 40^{\prime} \mathrm{W}\right)$, hacia el sector central $\left(78^{\circ} 30^{\prime} \mathrm{W}\right)$ y la parte sur $\left(79^{\circ} 35^{\prime} \mathrm{W}\right)$.

La segunda capa (0-30(50) metros) presenta una extensión de mas o menos $1^{\circ}$ pasando por el centro de la región de estudio desde el noroccidente hacia la región comprendida por los $79^{\circ}$ y $80^{\circ} \mathrm{W}$ y después hacia el sur entre $79^{\circ} 45^{\prime}$ y $81^{\circ} \mathrm{W}$ aproximadamente.

La tercera capa (0-50(75) metros), se encuentra en el sector sur occidental del Pacifico Colombiano ocupando el resto de la región de estudio.

En las profundidades mayores, se observa una división de la siguiente manera (Fig. No.5): las aguas costeras presentan tres diferentes regiones, la primera localizada desde los $4^{\circ} 30^{\prime} \mathrm{N}$ y más hacia al norte correspondiente a la capa de 200-400 metros, por este sector, se observa una pequeña región alrededor de la estación No.1 (Fig. No.1), donde no existe relación estadística entre las capas profundas. La se- gunda (desde los $4^{\circ} 30^{\prime} \mathrm{N}$ hacia el sur cerca de la costa, llegando a los $2^{\circ} 20^{\prime} \mathrm{N}$ ) carece de relaciones estadísticas. El resto de las aguas costeras del sur presenta relación estadística de la capa 100-500 metros. Esta región se extiende hacia el occidente hasta $\operatorname{los} 80^{\circ} 40^{\prime} \mathrm{W}$ y hacia el norte hasta los $3^{\circ} 40^{\prime} \mathrm{N}$. Esta misma capa se observa en el sector norte de la región y parte del occidente delimitando al Pacifico Colombiano.

La capa 150-500 metros se localiza en el resto de la región de estudio pasando por la parte central desde el sur occidente hacia el nororiente del Pacifico Colombiano.

De lo anterior, se observa que las aguas superficiales y "profundas" (hasta los 500 metros analizados) presentan entre sí una capa intermedia donde no existe una correlación dentro de la misma ni dependencia con respecto a las capas superficiales y profundas. Esta capa intermedia varia en grosor dependiendo de la región donde se encuentra y puede mostrar una ubicación aproximada en la capa de "salto" entre los valores de Temperatura y Salinidad denominadas termoclina y haloclina.

Los demás sectores presentados en las Figuras No.2, 3,4 y 5 con diversos colores, muestran en que lugares la Temperatura y la Salinidad poseen unas oscilaciones mensuales en un año similares o dependientes entre sí por capas o profundidades.

La Temperatura, sobre todo a lo largo de la zona costera presenta grandes cambios estacionales a través del año (Bubnov, 1990)

En el Pacifico Colombiano se observa que las capas superficiales más delgadas se encuentran en la zona costera y se puede decir que tienen un desarrollo leve de la capa superficial homogénea, ya que solamente el sector norte se presenta una capa homogénea hasta de 0-100(150) metros, donde se observan las corrientes provenientes del Golfo de Panamá las cuales pueden ocupar gran parte de las profundidades 0-100(150) metros.

La Salinidad del agua superficial (Fig. No.4) es conservativa y, por la dependencia de correlación entre las profundidades, se divide solamente en tres regiones. El sector de la capa más delgada (zona costera) presenta aguas de menor Salinidad debido a la cantidad de ríos que llegan al Pacifico Colombiano. Luego, esta agua se extiende al océano, donde encuentra aguas mas salinas formando una capa ho- 


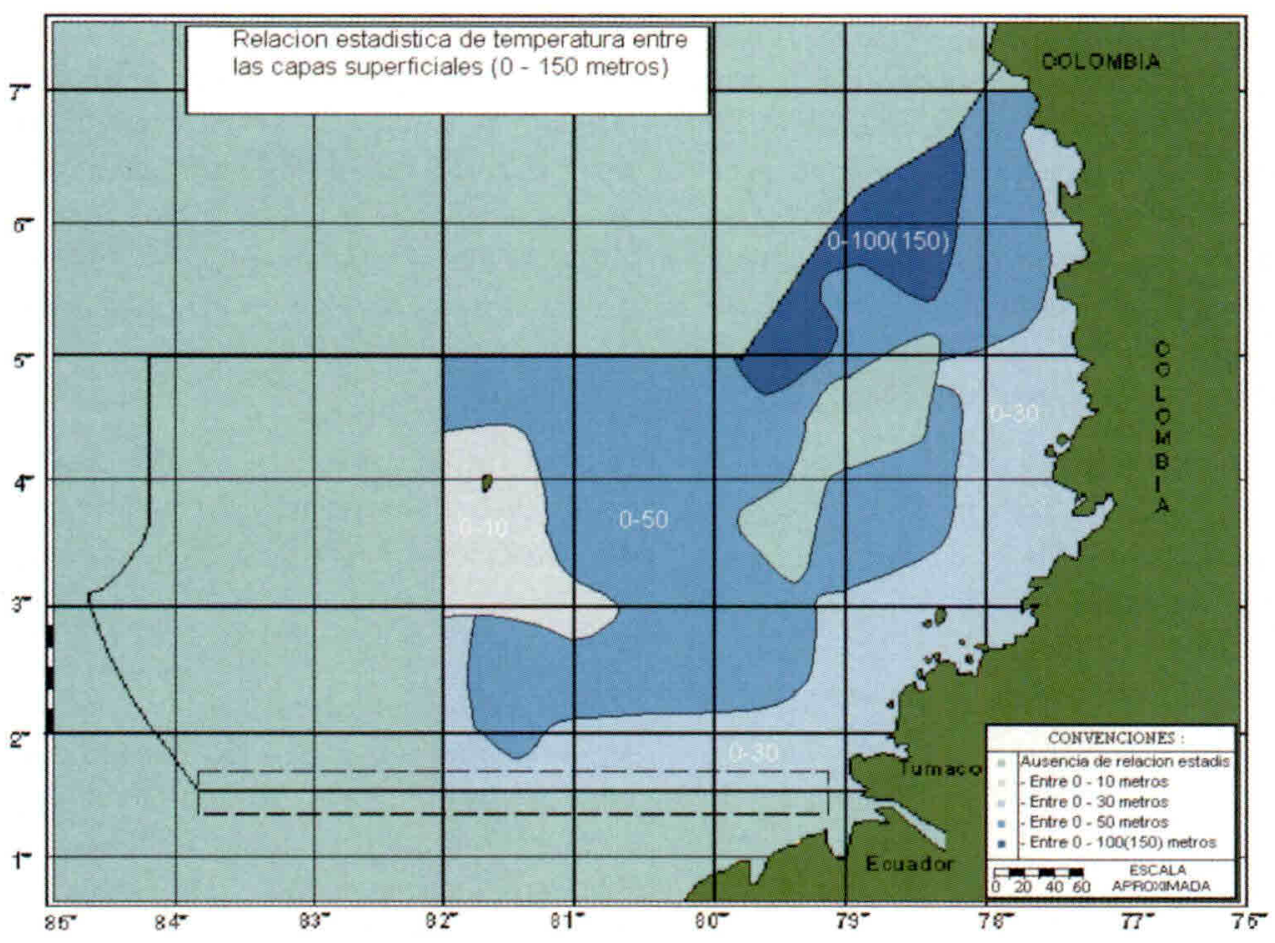

Figura 2

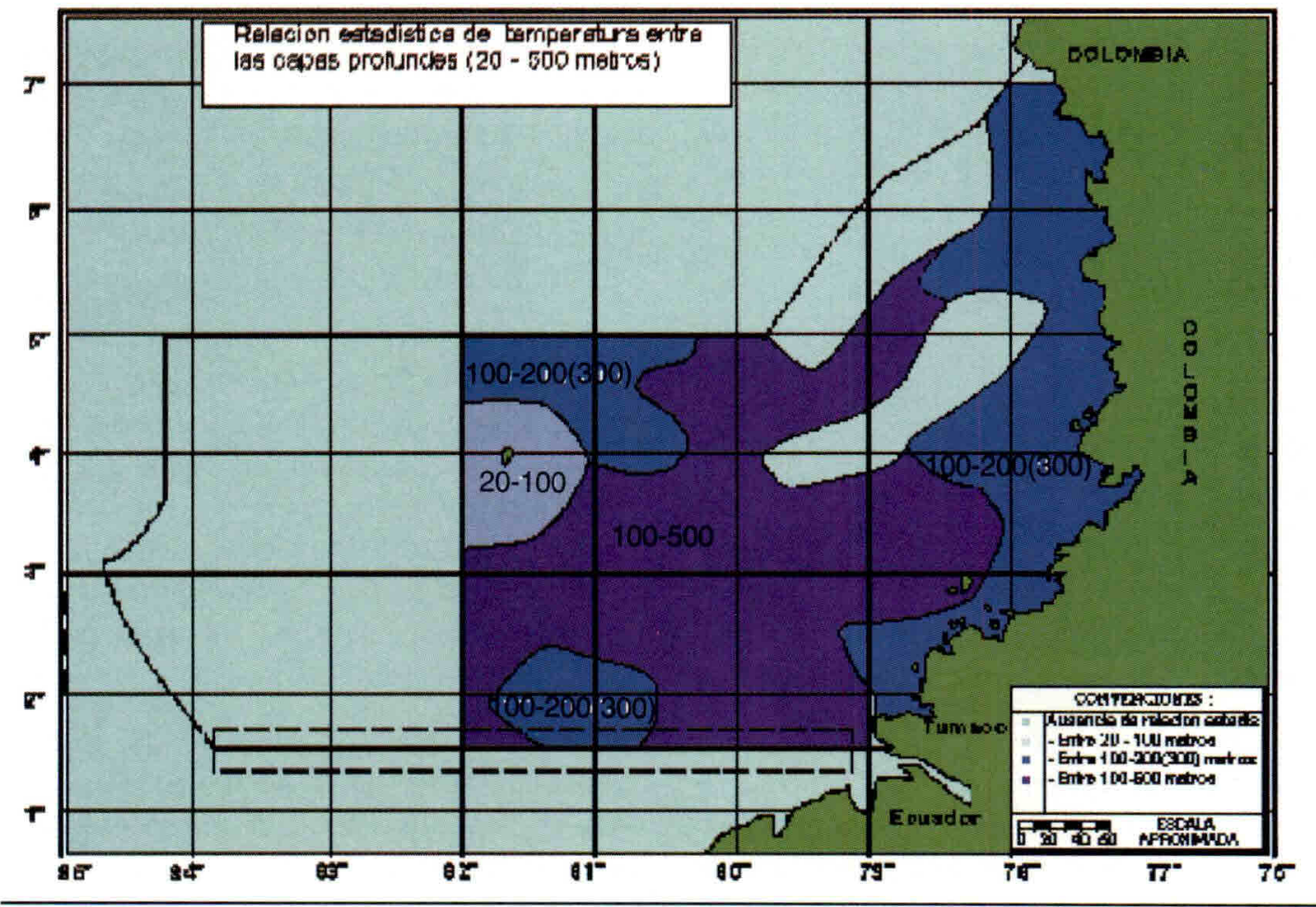

Figura 3 


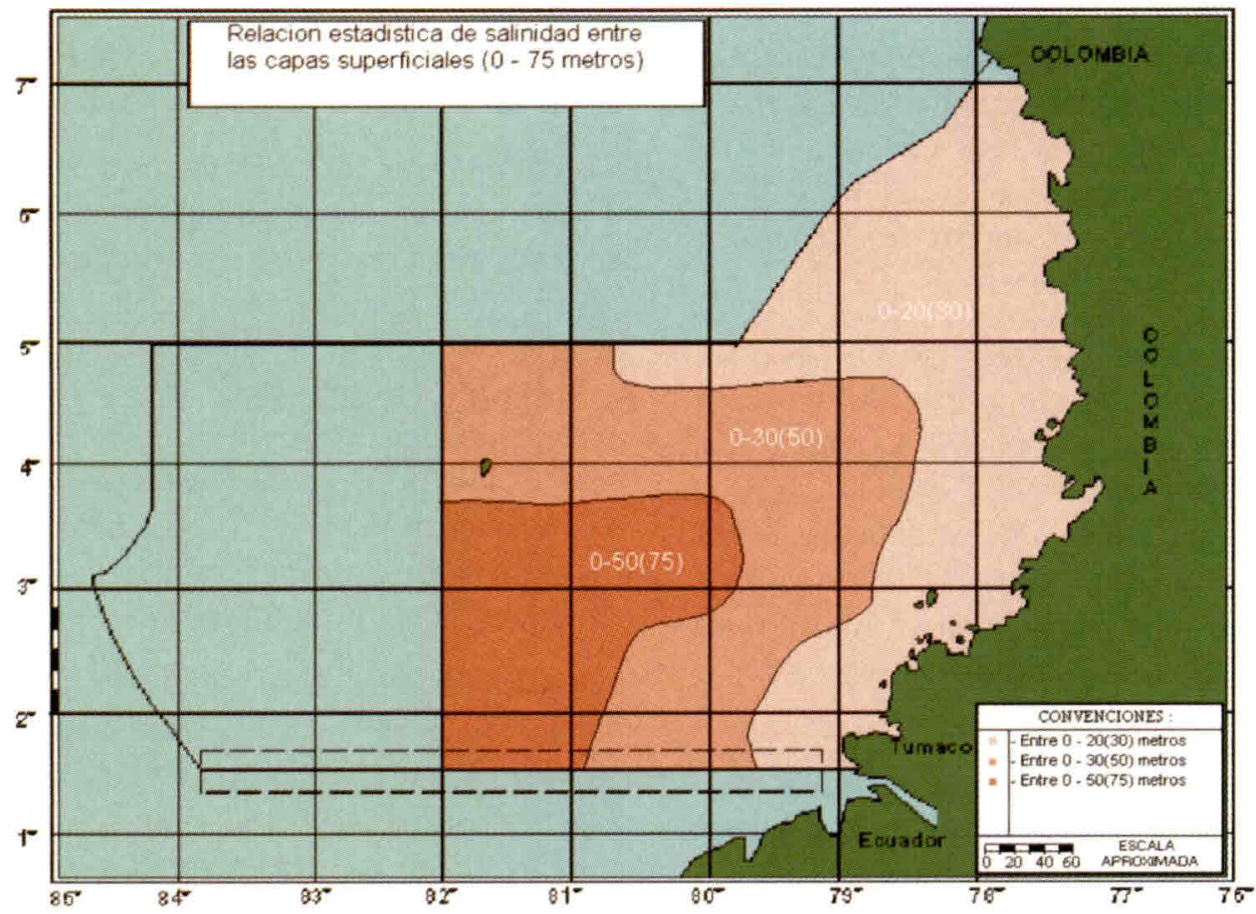

Figura 4

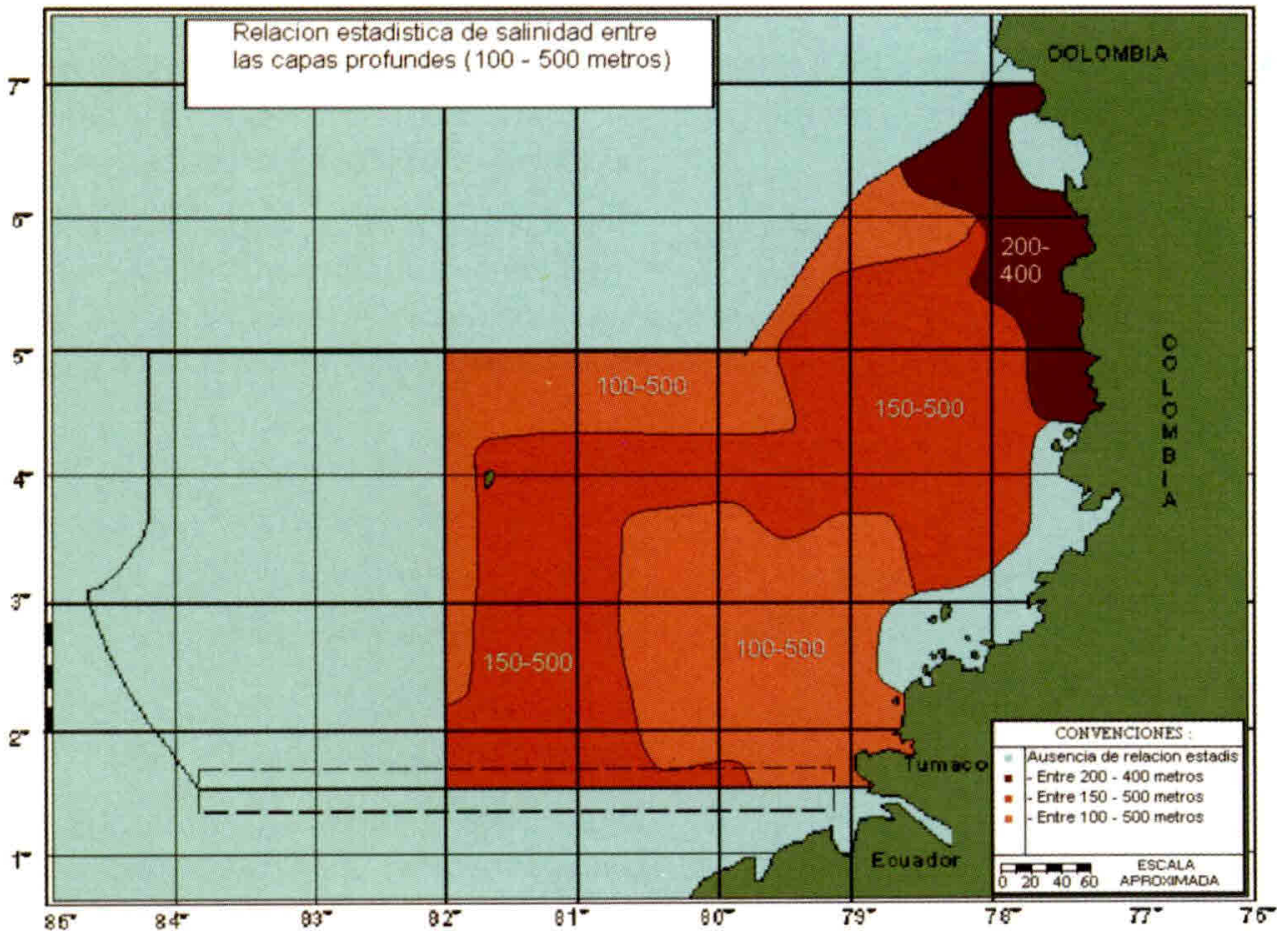

Figura 5 
mogénea hasta de 0-30(50) metros, la cual se constituye en él limite entre las aguas de menor Salinidad (aguas costeras) y las mas salinas (zona oceánica). Las aguas mas salinas forman una capa homogénea de 0-50(75) metros y se localizan al occidente de la región de estudio con una profundización de la capa de "salto" de la Salinidad (haloclina).

En las aguas "profundas", con respecto a la Temperatura (Fig. No.3) se observa que por debajo de la termoclina existe una capa homogénea más gruesa que la de las aguas superficiales ya que esta agua posee un gradiente vertical de Temperatura menor que el de las superficiales. Las aguas costeras son más delgadas debido a los movimientos verticales, los cuales cambian la estructura de las aguas localizadas por debajo de la capa 100-200(300) metros.

Se observan dos pequeñas sectores donde no existen relaciones estadísticas de correlación entre las profundidades debido a la complejidad de los movimientos dinámicos, donde ocurre la mezcla de aguas de dos corrientes: la del golfo de Panamá y la que surge entre la corriente Colombiana y la proveniente del occidente del Pacifico Colombiano.

Acerca de la Salinidad de las aguas profundas se puede decir, que debido al movimiento vertical de las aguas no se encuentra una dependencia clara entre las zonas costeras, pero en la zona oceánica si se logra obtener una capa homogénea de correlación estadística entre las profundidades.

Los resultados obtenidos no se pueden considerar ciertos en su totalidad, ya que para conseguir él calculo de los coeficientes de correlación se utilizaron las características medias mensuales de Temperatu- ra y Salinidad obtenidas a través de una insuficiente cantidad de datos iniciales, lo cual disminuye la veracidad de los valores medios mensuales.

De todas maneras, los resultados presentados en este trabajo muestran una visión aproximada de las dependencias estadísticas entre las profundidades analizadas para la Temperatura y la Salinidad.

\section{CONCLUSIONES}

Los resultados presentados en este trabajo muestran una visión aproximada de las dependencias estadísticas entre las profundidades analizadas con respecto a la Temperatura y la Salinidad.

Se determino que el desarrollo de la capa superficial homogénea en el Pacífico Colombiano es leve, ya que solamente el sector norte presenta una capa homogénea hasta de 0-100(150) metros. Se observó que las capas superficiales más delgadas se encuentran en la zona costera.

La Salinidad del agua superficial del Pacífico Colombiano es conservativa y, por la dependencia de correlación entre las profundidades, se divide en tres regiones: zona costera, zona oceánica y una zona entre ellos (con las capas 0-20, 0-30(50), 0-50(75) metros respectivamente).

En las aguas "profundas", por debajo de la termoclina, existe una capa homogénea más gruesa que la de las aguas superficiales y en algunas zonas esta capa no existe.

En la Salinidad de las aguas profundas no se encuentra una dependencia clara entre las zonas costeras, pero en la zona oceánica si se logró obtener una capa homogénea de correlación estadística entre las profundidades.

\section{Bibliografía}

ABUZIAROV, Z.K. Pronósticos Marinos. Gidrometeoizdat, Leningrado, 1988.

ANDRADE, C.A. Movimiento Geostrófico en el Pacífico Colombiano. Boletín Científico ClOH No.12. 23 - 38. Cartagena, 1992.

BUBNOV, V.A. Circulación de las Aguas de la Zona Ecuatorial del Océano Mundial. Gidrometeoizdat, Leningrado, 1990.
DORONIN, Y.P. Dinámica del Océano. Gidrometeoizdat, Leningrado, 1980.

DORONIN, Y.P. Oceanología Regional. Gidrometeoizdat, Leningrado, 1986.

FEODOROV, K.N. Cambios Globales en el Régimen de Interacción Océano Atmósfera y EL NIÑO 1982 1983. Colección de Trabajos. pp. 162 - 173. Gidrometeoizdat, Leningrado, 1990. 
KAGAN, B.A. Interacción Océano Atmósfera. Gidrometeoizdat, San Petersburgo, 1992.

KRAUS, E.B. and BUSINGER, J.A. Atmosphere Ocean Interaction. Oxford Monographs On Geology And Geophysics No.27. Oxford University Press, New York, 1994.

MÁLIKOV, I. Búsqueda de los Cambios mensuales en un año de Temperatura y Salinidad del Pacífico Colombiano. Informe Final., 1998.

MILLAN, E. y BEJARANO, J. Pacífico Colombiano 1989 Condiciones Termohalinas y Estandarización de la Curva T-S. Boletín Científico ClOH No.15. 39 - 65. Cartagena, 1994.
PINEDA, A.R. y VILLEGAS, N.L. Condiciones Hidrológicas y Biológicas en el Pacífico Colombiano y en la Ensenada de Tumaco Durante el Período de 1996. Reporte Final. Tumaco-Colombia, 1996.

VAINOVSKY, P.A. y MALININ, V.N. Métodos del Tratamiento y Análisis de la Información Oceanológica. Gidrometeoizdat, Leningrado, 1991.

VILLEGAS, N.L. Estudio del Movimiento Vertical de las Aguas en la Región Este de la Cuenca del Pacifico Colombiano. Tesis de Pregrado. San Petersburgo), 1995. 